\title{
Association of human epidermal growth factor receptor 2 status with ipsilateral breast tumor recurrence and resistance to endocrine therapy
}

\author{
MAKOTO ISHITOBI, MIZUHO SHIBA, TAKAHIRO NAKAYAMA, HIROKI KOYAMA and YASUHIRO TAMAKI
}

Department of Breast and Endocrine Surgery, Osaka Medical Center for Cancer

and Cardiovascular Diseases, Osaka, Osaka 537-8511, Japan

Received February 6, 2015; Accepted March 20, 2015

DOI: $10.3892 / \mathrm{mco} .2015 .534$

\begin{abstract}
The effect of prior endocrine therapy on tumor biology and clinical outcomes of locoregional recurrence remains unclear. A total of 76 patients, who underwent salvage breast surgery for estrogen receptor-positive ipsilateral breast tumor recurrence (IBTR) following breast-conserving surgery for primary breast cancer, were retrospectively reviewed to investigate the association of human epidermal growth factor receptor 2 (HER2) status with the characteristics of IBTR and clinical outcomes following resection of IBTR. There was a tendency for more patients with HER2-positive IBTR to show resistance to endocrine therapy $(71.4 \%)$ compared with those with HER2-negative IBTR $(37.3 \%)(\mathrm{P}=0.085)$. Patients with HER2-positive IBTR exhibited a higher rate of recurrence (62.5\%) compared with those with HER2-negative IBTR $(25.0 \%)(\mathrm{P}=0.027)$. These results suggested that HER2-positive IBTR may be associated with resistance to endocrine therapy and a poorer clinical outcome.
\end{abstract}

\section{Introduction}

Breast-conserving surgery is the standard treatment for early breast cancer. However, $\sim 5-10 \%$ of the patients treated with breast-conserving surgery subsequently develop ipsilateral breast tumor recurrence (IBTR) within 10 years $(1,2)$. IBTR following breast-conserving treatment is associated with an increased risk of distant disease and mortality (3-6). The available data supporting the recommendation of systemic treatment for breast cancer patients with locoregional

Correspondence to: Dr Makoto Ishitobi, Department of Breast and Endocrine Surgery, Osaka Medical Center for Cancer and Cardiovascular Diseases, 1-3-3 Nakamichi, Osaka, Osaka 537-8511, Japan

E-mail: isitobi-ma@mc.pref.osaka.jp

Key words: breast cancer, ipsilateral breast tumor recurrence, endocrine therapy, resistance recurrence are sparse (7-9), whereas adjuvant systemic therapy reduces the risk of relapse and mortality in primary breast cancer patients $(10,11)$. To the best of our knowledge, there has been only one randomized controlled study assessing whether adjuvant endocrine therapy reduces the rate of recurrence in breast cancer patients with locoregional recurrence; the Swiss Group for Clinical Cancer Research demonstrated that tamoxifen following local treatment significantly improved the disease-free survival (DFS) of breast cancer patients with locoregional recurrence (8). However, patients pretreated with adjuvant tamoxifen were not eligible for that study. Therefore, it is unknown whether endocrine therapy may effectively decrease the incidence of recurrence in breast cancer patients with locoregional recurrence who have received adjuvant endocrine therapy for the primary tumor.

In clinical practice, the estrogen receptor (ER) and human epidermal growth factor receptor 2 (HER2) statuses were found to be factors essential for guiding therapeutic decision-making regarding endocrine and anti-HER2 therapy. Preclinical studies investigated the crosstalk between ER and HER 2 and its effect on resistance to endocrine therapy (12-14). In this study, we aimed to assess the association of the HER2 status of ER-positive IBTR following breast-conserving treatment with prior endocrine therapy and patient outcomes.

\section{Patients and methods}

Patient selection. We reviewed the records of 3,793 breast cancer patients who underwent breast-conserving surgery between 1989 and 2013 at the Department of Breast and Endocrine Surgery, Osaka Medical Center for Cancer and Cardiovascular Diseases (Osaka, Japan). A total of 180 patients $(4.7 \%)$ developed IBTR as a first event, without evidence of synchronous metastatic disease, and underwent salvage surgery. Following the exclusion of i) patients who received neoadjuvant therapy as part of the initial treatment regimen, ii) patients with non-invasive tumors in IBTR specimens and iii) patients who underwent salvage surgery for IBTR prior to 2000 (due to lack of information regarding HER2 status), there were a total of 116 patients with IBTR for whom information was available regarding ER and HER2. Of these 116 patients, 76 with ER-positive IBTR were finally included in this analysis. 
Table I. Patient characteristics.

\begin{tabular}{|c|c|c|c|c|}
\hline Characteristics & All $(n=76)$ & HER2-negative $(\mathrm{n}=68)$ & HER2-positive ( $\mathrm{n}=8$ ) & P-value \\
\hline Age at initial diagnosis, years & & & & 0.78 \\
\hline Median (range) & $49(29-81)$ & $49(30-81)$ & $50(29-69)$ & \\
\hline $\begin{array}{l}\text { Adjuvant chemotherapy } \\
\text { following primary surgery }\end{array}$ & & & & 0.45 \\
\hline Yes & 20 & 17 & 3 & \\
\hline No & 56 & 51 & 5 & \\
\hline $\begin{array}{l}\text { Adjuvant endocrine therapy } \\
\text { following primary surgery }\end{array}$ & & & & 0.43 \\
\hline Yes & 58 & 51 & 7 & \\
\hline No & 18 & 17 & 1 & \\
\hline $\begin{array}{l}\text { Adjuvant trastuzumab } \\
\text { following primary surgery }\end{array}$ & & & & NE \\
\hline Yes & 0 & 0 & 0 & \\
\hline No & 76 & 68 & 8 & \\
\hline
\end{tabular}

Time interval from initial surgery to IBTR, years Median (range)

Age at IBTR, years Median (range)

pT stage of recurrent breast tumor

Unknown

breast tumor

$\begin{array}{lr}1 & 17 \\ 2 & 34 \\ 3 & 16 \\ \text { Unknown } & 9\end{array}$

Lymphovascular invasion of recurrent breast tumor
Negative
Positive

Unknown

Histological type of recurrent breast tumor Invasive ductal

Other

Unknown

Adjuvant chemotherapy following salvage surgery

Adjuvant endocrine therapy following salvage surgery 
Table I. Continued.

\begin{tabular}{lcccr}
\hline Characteristics & All $(\mathrm{n}=76)$ & HER2-negative $(\mathrm{n}=68)$ & HER2-positive $(\mathrm{n}=8)$ & P-value \\
\hline Any recurrence & & & & 0.027 \\
Absent & 54 & 51 & 3 & \\
Present & 22 & 17 & 5 & \\
\hline
\end{tabular}

IBTR, ipsilateral breast tumor recurrence; HER2, human epidermal growth factor receptor 2; NE, not evaluable.

This study was approved by the local Ethics Committee of Osaka Medical Center of Cancer and Cardiovascular Diseases.

Hormone receptor status. The patients received a physical examination every 3-6 months for the first 5 years following primary and salvage surgery and annually thereafter. Mammograms were performed annually following primary and salvage surgery. The ER status was determined by immunohistochemistry and tumors with $\geq 10 \%$ positively stained cells were classified as positive. The HER2 status was considered as positive if immunohistochemistry was $3+$ or if the fluorescence in situ hybridization amplification score (HER2/neu to chromosome 17 ratio) was $>2.0$.

Definition of resistance. Resistance to endocrine therapy was defined as disease relapse while receiving adjuvant endocrine therapy, or relapse within 12 months of completing such therapy (15). The absence of resistance to endocrine therapy was defined as relapse after 12 months of completing adjuvant endocrine therapy.

Statistical analysis. The association of the HER2 status with various clinical or pathological factors of IBTR was assessed using the Student's t-test or the Chi-square test. DFS was defined as the period from the date of surgery for IBTR to the date of appearance of any recurrence and was calculated by the Kaplan-Meier method. The log-rank test was used to evaluate differences in DFS among various patient subgroups. All the statistical tests and $\mathrm{P}$-values were two-tailed and $\mathrm{P}<0.05$ was considered to indicate a statistically significant difference. All the statistical analyses were performed with SPSS statistical software, version 21 (IBM Japan, Ltd., Tokyo, Japan).

\section{Results}

Patient characteristics and association with HER2 status. The patient characteristics according to the HER2 status are shown in Table I. With respect to clinicopathological factors, there were no significant differences between patients with HER2-positive and those with HER2-negative IBTR, except in patients with lymphovascular invasion $(\mathrm{P}=0.024)$.

Of the 76 patients, 58 (76.3\%) received adjuvant endocrine therapy following primary surgery. Among these 58 patients, there was a non-significant tendency for more patients with HER2-positive IBTR to show resistance to endocrine therapy $(71.4 \%, 5 / 7)$ compared with those with HER2-negative IBTR $(37.3 \%, 19 / 51)(\mathrm{P}=0.085$ by Chi-square test; data not shown).
Follow-up. Within a median follow-up period of 2.4 years (range, 0.1-11.6 years), 22 of the 76 patients (28.9\%) experienced disease recurrence. Patients with HER2-positive IBTR exhibited a higher rate of recurrence (62.5\%) compared with those with HER2-negative IBTR (25.0\%) (Chi-square $\mathrm{P}=0.027$; Table I). The 3 -year DFS rates in patients with HER2-positive and -negative IBTR were 50.0 and $77.0 \%$, respectively (log-rank test $\mathrm{P}=0.34$; data not shown).

\section{Discussion}

In this study, the presence of resistance to endocrine therapy was associated with a higher frequency of HER2-positive IBTR. Moreover, patients with HER2-positive IBTR exhibited a higher rate of recurrence compared with those with HER2-negative IBTR. Our analysis was limited to patients with ER-positive IBTR who were candidates for endocrine therapy following IBTR resection. There is limited available evidence regarding the effect of adjuvant endocrine therapy on decreasing recurrence in patients with IBTR following its resection. Our findings may provide a new insight regarding the mechanisms of resistance to endocrine therapy.

Several preclinical studies have investigated the crosstalk between ER and HER2 and the effect of the HER2 status on resistance to endocrine therapy (12-14). Massarweh et al (14) reported that acquired tamoxifen resistance in MCF-7 tumors was associated with increased HER2 expression, which was consistent with our clinical data. Until recently, the available clinical data on the association between biomarker changes and resistance to endocrine therapy have been limited. Arnedos et al (16) investigated biomarker changes associated with the development of resistance to aromatase inhibitors in ER-positive breast cancer. The authors of that study evaluated biomarker changes using pairs of samples from patients who had relapsed or progressed while receiving aromatase inhibitors and found that there were no marked changes in the HER2 status; however, in a small proportion of tumors $(5.6 \%, 3 / 54)$, HER 2 was positive in recurrent tumors, despite the primary tumors being HER2-negative.

Several studies reported on the association between prior systemic therapy and the characteristics of subsequently developing tumors, including contralateral breast cancer (17) or any site of recurrence (18). Swain et al (17) reported that patients with ER-positive primary cancer who received tamoxifen had fewer ER-positive contralateral breast cancers compared with those who did not receive tamoxifen, which may be a result of tamoxifen treatment. In that study, analyses of the HER2 
status were not performed. Niikura et al (18) evaluated the HER2 status of metastatic sites in patients with HER2-positive primary tumors and found that HER2-negative metastatic tumors were more frequent among patients who had received chemotherapy compared with those who had not. Prior trastuzumab did not affect the HER2 status of metastatic tumors. The results suggested that the increased rate of HER2-negative metastatic tumors following chemotherapy may be associated with biopsies sampling HER2-negative subclones.

Our study had several limitations, including the small sample size, short follow-up period, retrospective nature of the study design and lack of information regarding primary tumors. In addition, as the majority of the patients in our study received treatment for primary tumors in the pre-trastuzumab era, the effect of prior trastuzumab on the breast cancer subtype of the recurrent tumors is unknown.

In conclusion, the HER2 status of IBTR was found to be associated with resistance to endocrine therapy and a poorer clinical outcome. However, further investigation is required to elucidate the mechanisms of resistance to endocrine therapy.

\section{Acknowledgements}

This study was supported in part by the Osaka Foundation for the Prevention of Cancer and Cardiovascular Diseases.

\section{References}

1. Fisher B, Anderson S, Bryant J, Margolese RG, Deutsch M, Fisher ER, Jeong JH and Wolmark N: Twenty-year follow-up of a randomized trial comparing total mastectomy, lumpectomy and lumpectomy plus irradiation for the treatment of invasive breast cancer. N Engl J Med 347: 1233-1241, 2002.

2. Veronesi U, Cascinelli N, Mariani L, Greco M, Saccozzi R, Luini A, Aguilar M and Marubini E: Twenty-year follow-up of a randomized study comparing breast-conserving surgery with radical mastectomy for early breast cancer. N Engl J Med 347: 1227-1232, 2002.

3. Komoike Y, Akiyama F, Iino Y, et al: Ipsilateral breast tumor recurrence (IBTR) after breast-conserving treatment for early breast cancer: Risk factors and impact on distant metastases. Cancer 106: 35-41, 2006.

4. Anderson SJ, Wapnir I, Dignam JJ, Fisher B, Mamounas EP, Jeong JH, Geyer CE Jr, Wickerham DL, Costantino JP and Wolmark N: Prognosis after ipsilateral breast tumor recurrence and locoregional recurrences in patients treated by breast-conserving therapy in five National Surgical Adjuvant Breast and Bowel Project protocols of node-negative breast cancer. J Clin Oncol 27: 2466-2473, 2009.

5. Whelan T, Clark R, Roberts R, Levine M and Foster G; Investigators of the Ontario Clinical Oncology Group: Ipsilateral breast tumor recurrence postlumpectomy is predictive of subsequent mortality: Results from a randomized trial. Int J Radiat Oncol Biol Phys 30: 11-16, 1994.
6. Haffty BG, Reiss M, Beinfield M, Fischer D, Ward B and McKhann C: Ipsilateral breast tumor recurrence as a predictor of distant disease: Implications for systemic therapy at the time of local relapse. J Clin Oncol 14: 52-57, 1996.

7. Rauschecker H, Clarke M, Gatzemeier W and Recht A: Systemic therapy for treating locoregional recurrence in women with breast cancer. Cochrane Database Syst Rev 4: CD002195, 2001.

8. Waeber M, Castiglione-Gertsch M, Dietrich D, Thürlimann B, Goldhirsch A, Brunner KW and Borner MM; Swiss Group for Clinical Cancer Research (SAKK): Adjuvant therapy after excision and radiation of isolated postmastectomy locoregional breast cancer recurrence: Definitive results of a phase III randomized trial (SAKK 23/82) comparing tamoxifen with observation. Ann Oncol 14: 1215-1221, 2003.

9. Aebi S, Gelber S, Anderson SJ, et al; CALOR investigators: Chemotherapy for isolated locoregional recurrence of breast cancer (CALOR): A randomised trial. Lancet Oncol 15: 156-163, 2014.

10. Davies C, Godwin J, Gray R, et al; Early Breast Cancer Trialists Collaborative Group (EBCTCG): Relevance of breast cancer hormone receptors and other factors to the efficacy of adjuvant tamoxifen: Patient-level meta-analysis of randomised trials. Lancet 378: 771-784, 2011.

11. Peto R, Davies C, Godwin J, et al; Early Breast Cancer Trialists Collaborative Group (EBCTCG): Comparisons between different polychemotherapy regimens for early breast cancer: Meta-analyses of long-term outcome among 100,000 women in 123 randomised trials. Lancet 379: 432-444, 2012.

12. Knowlden JM, Hutcheson IR, Jones HE, Madden T, Gee JM, Harper ME, Barrow D, Wakeling AE and Nicholson RI: Elevated levels of epidermal growth factor receptor/c-erbB2 heterodimers mediate an autocrine growth regulatory pathway in tamoxifen-resistant MCF-7 cells. Endocrinology 144: 1032-1044, 2003.

13. Shou J, Massarweh S, Osborne CK, Wakeling AE, Ali S, Weiss H and Schiff R: Mechanisms of tamoxifen resistance: Increased estrogen receptor-HER2/neu cross-talk in ER/HER2-positive breast cancer. J Natl Cancer Inst 96: 926-935, 2004.

14. Massarweh S, Osborne CK, Creighton CJ, Qin L, Tsimelzon A, Huang S, Weiss H, Rimawi M and Schiff R: Tamoxifen resistance in breast tumors is driven by growth factor receptor signaling with repression of classic estrogen receptor genomic function. Cancer Res 68: 826-833, 2008.

15. Cardoso F, Costa A, Norton L, et al: ESO-ESMO 2nd international consensus guidelines for advanced breast cancer (ABC2). Breast 23: 489-502, 2014.

16. Arnedos M, Drury S, Afentakis M, A'Hern R, Hills M, Salter J, Smith IE, Reis-Filho JS and Dowsett M: Biomarker changes associated with the development of resistance to aromatase inhibitors (AIs) in estrogen receptor-positive breast cancer. Ann Oncol 25: 605-610, 2014.

17. Swain SM, Wilson JW, Mamounas EP, Bryant J, Wickerham DL, Fisher B, Paik S and Wolmark N: Estrogen receptor status of primary breast cancer is predictive of estrogen receptor status of contralateral breast cancer. J Natl Cancer Inst 96: 516-523, 2004.

18. Niikura N, Liu J, Hayashi N, Mittendorf EA, Gong Y, Palla SL, Tokuda Y, Gonzalez-Angulo AM, Hortobagyi GN and Ueno NT: Loss of human epidermal growth factor receptor 2 (HER2) expression in metastatic sites of HER2-overexpressing primary breast tumors. J Clin Oncol 30: 593-599, 2012. 